\title{
DINÁMICA DE LOS COMPONENTES DEMOGRÁFICOS EN BAJA CALIFORNIA, DURANTE EL PERÍODO 1985-1990*
}

\author{
por \\ Gabriel Estrella Valenzuela**
}

\begin{abstract}
RESUMEN
En este trabajo se identifican los rangos predominantes en la dinámica de los componentes demográficos del estado de Baja California y sus municipios, durante el período 1985-1990. Para ello, se utilizan y combinan datos de estadísticas vitales, censales y de las Encuestas Demográficas de Baja California de 1986 y 1990.

A partir del análisis de la mortalidad y la fecundidad, se logra determinar una tendencia de estabilidad en el componente natural de la dinámica demográfica del estado, con variaciones marginales a nivel municipal. En el rubro de crecimiento social, sin embargo, se observa un notable y claro incremento en la movilidad de la población que se sustenta en dos componentes: un incremento en la tasa de emigración desde el estado en su conjunto y una más elevada y consistente tendencia al incremento en las tasas de inmigración hacia Baja California, que revierte la tendencia decreciente del peso del crecimiento social en la entidad.
\end{abstract}

\begin{abstract}
Combining data from vital statistics, census and the 1986 and $1990 \mathrm{Baja}$ California Demographic Surveys, in this paper we analyze the main characteristics of the demographic variables for the State of Baja California and its municipalities, during the 1985-1990 period.

Through a mortality and fertility analysis, it is established a stability trend in the natural component of the demographic dynamic at the State level with only marginal variations for the four municipalities. In regard to social growth, however, there is a clear and noticeable increase in the State's population mobility sustained on two components: a trend of growing rates of emigration from all over the State, and a still higher trend of increases of the immigration rates for the State as a whole, which reverts a tendency of decreasing importance of social growth for the state.
\end{abstract}

- Ponencia presentada en el evento "A dos años del XI Censo. Taller sobre población en Baja Califomia y sus municipios"; organizado por el Centro de Estudios para el Desarrollo de la Adminústración Municipal, A. C.

** Investigador del Instituto de Investigaciones Sociales, UABC. 


\section{INTRODUCCIÓN}

En un número reciente de la revista DEMOS (1991), aparece un artículo atribuido a un alıo funcionario de INEGI (Cervera:1991) en el que se afirma que,

...la población calculada por el censo de 1980...por problemas de cobertura (se) cuantificó con base en la aplicación de técnicas complementarias. A la luz de los resultados del censo de 1990 es factible estimar la escasa bondad de las técnicas ...(pues)... hemos podido conocer que se sobrestimaron las cifras, nacional y de algunas entidades federativas... y se subestimaron (las de otras) (Ibid;39. S.N.).

Esta revelación, que por una parte reivindica a quienes por algún tiempo hemos insistido en la necesidad de diseñar e implementar "técnicas" paralelas y complementarias a las censales para hacer estimaciones demográficas en períodos intercensales, también se convierte en un llamado de atención para efectuar una lectura cautelosa de las tendencias de los componentes demográficos, especialmente cuando éstas se estiman sólo en base a "cuantificaciones" censales de los volúmenes de población.

Buscando estimar dichas tendencias a partir de fuentes complementarias y actualizadas, el objetivo central de este documento consiste en presentar las características predominantes en la dinámica de los componentes demográficos del estado de Baja California y sus municipios, durante el período 1985-1990.

Para el logro de dicho propósito, en el primer apartado de esta presentación se hace una breve recapitulación de la trayectoria demográfica de Baja California hasta 1980. A continuación, utilizando datos recientes procedentes de cuatro fuentes (i.e. Las estadísticas vitales; el XI Censo General de Población y Vivienda de 1990, y; las Encuestas Demográficas de Baja California de 1986 y 1990), y recurriendo a un procedimiento propuesto por R. Corona (1991), se describe el comportamiento de algunos de los componentes de la dinámica demográfica de la entidad y sus municipios durante el período 1985-1990 para, finalmente, exponer en la tercer sección las conclusiones que de ello se derivan.

\section{ANTECEDENTES DEMOGRÁFICOS DE BAJA CALIFORNIA}

El estado de Baja California presenta una dinámica poblacional muy acelerada con respecto a la forma en que el total del pais ha evolucionado durante las primeras ocho décadas del presente siglo. Durante la mayor parte de ese período, el estado muestra ritmos de crecimiento sistemáticamente más dinámicos que los del total de la nación. 
A manera de ilustración, baste señalar que el nivel de las tasas de crecimiento total (TCT) de la población del estado, resultó superior al observado en el total de la población del país para las décadas 1940-1950, 1950-1960 y 1960-1970, pues mientras México crecía a tasas de 2.7, 3.1 y $3.4 \%$ anual, Baja California crecía a tasas del $10.9,8.6$ y $5.5 \%$ anual en los mismos períodos.

Sin embargo, durante este período la tendencia de la TCT de la población del estado fue de paulatina pero constante reducción. De esta forma, para el período 1970-1980 el ritmo de crecimiento anual de la población total de Baja California, era ya de $3.0 \%$, lo que implicó una reducción de casi 35.0\% en relación a la tasa observada en el período 1960-1970.

La distribución de la población a nivel municipal ha variado a través del tiempo. En 1930, la mayor parte de la población (62.0\%) se encontraba residiendo en Mexicali, cuya cabecera municipal es la capital del estado, y un poco más de $23.0 \%$ en Tijuana. A partir de ese año el municipio de Tijuana, que cuenta con el territorio de menor tamaño, ha tendido a poblarse más relativamente.

El municipio de Ensenada - el más extenso territorialmente hablando- no ha sufrido grandes variaciones desde 1930, contribuyendo desde entonces con alrededor de $13.0 \%$ a la población total de la entidad. Tecate por su parte, permanece siendo el municipio más pequeño en cuanto a monto de población se refiere; desde el año 1950, en que fue censado por primera vez como municipio autónomo, ha contribuido con alrededor de $2.0 \%$ a la población total del estado.

En forma similar a lo observado en el caso de la entidad en su conjunto, en los últimos decenios del período señalado se empieza a observar que los ritmos de crecimiento de los cuatro municipios de la entidad se hacen más lentos. Tijuana experimentó aún una tasa de crecimiento total de $7.8 \%$ entre 1960 y 1970 , pero de 1970 a 1980 la tasa fue de $3.0 \%$; en el caso de Mexicali, el crecimiento fue de $3.6 \%$ anual entre 1960 y 1970 y de $2.5 \%$ en el período 1970-1980. Tecate, a pesar de ser el municipio más pequeño en cuanto a población se refiere, alcanzó en 1960-1970 un ritmo de crecimiento de $8.6 \%$ y en 1970-1980 de 5.2\%, el cual fue el más rápido en la entidad durante ésta última década.

$\mathrm{Al}$ analizar los componentes del crecimiento poblacional, es posible señalar que Baja California aparentemente se puede ubicar en la última etapa en el proceso de transición demográfica.

Al respecto, en términos de fecundidad, observamos que la natalidad, después de haberse mantenido en niveles muy altos por varias décadas (i.e. la tasa bruta de natalidad [TBN] se mantuvo por arriba de $45.0^{\circ}$ oo hasta 1965 ), en 1970 muestra un claro descenso al alcanzar un 
valor de $41.7 \%$ oo; en 1975 este indicador se redujo a un valor de $30.0 \%$, y para 1980 parece haberse ubicado en una tasa de $25.0 \%$ oo.

Paralelamente, en Baja California la mortalidad se ha caracterizado por presentar niveles comparativamente bajos desde 1950. En la siguiente década, sin embargo, esta variable demográfica prosiguió su descenso y para 1960 la tasa bruta de mortalidad (TBM) fue un poco superior a 8 por mil, lo cual la ubicó por abajo del promedio nacional que estaba entonces por arriba de 10 por mil habitantes.

En el transcurso de los siguientes veinte años, la tendencia iniciada durante la década de los cincuenta en materia de mortalidad se ha sostenido, y también se puede observar un aparente y relativo aceleramiento en el ritmo de reducción de la TBM. Los valores de este indicador en la entidad fueron de $7.6 \%, 6.4 \%$ y $6.5 \%$ en los años 1970, 1976 y 1978 respectivamente. Para el período 19701980 en su conjunto, este indicador se ha estimado en el orden de $6.2 \%$ (Corona, 1991:22).

Consecuentemente, la esperanza de vida al nacer - que es un indicador del promedio de años de vida que vivirán los individuos si se mantuvieran las mismas condiciones de vida del año de referencia - fue de más de 65 años en 1970, y desde esa fecha se ha sostenido por arriba del nivel alcanzado en este rubro por el país en su conjunto.

Finalmente, al abordar la tercera variable demográfica, se puede partir de la afirmación de que la migración interna puede ser considerada como el componente demográfico que ha determinado el modelo de crecimiento poblacional en el estado de Baja California. Partiendo de estimaciones realizadas por el método indirecto del índice de sobrevivencia censal, la migración neta intercensal del estado de Baja California fue positiva desde la década 1930-1940; los montos ascendieron hasta 122,769 personas en la década 1950-1960; y en la siguiente éstos descendieron a 118 mil. Es muy posible que esta tendencia a la disminución de los saldos netos migratorios haya continuado en la siguientes décadas, como lo muestra la tasa de crecimiento social (TCS) del período 1970-1980 (18.3\%o).

Así, a partir de los datos de las tasas de crecimiento social (TCS) del período 1970-1980, el estado en su conjunto puede ser incluido en la categoria de "débil atracción migratoria" (TCS de 1.0\%). Sin embargo, a nivel municipal, mientras que la categoría migratoria de Ensenada y de Mexicali es de "débil atracción migratoria" (TCS de $0.7 \%$ y $0.6 \%$, respectivamente), Tecate y Tijuana se consideran de fuerte atracción migratoria, por presentar TCS de 1.9 y $1.5 \%$ respectivamente.

La tendencia general en los saldos netos migratorios ha tenido como consecuencia una modificación en la categoría migratoria del estado. Para 
explicar dicha modificación es necesario mencionar al menos uno de los elementos que influyeron en el cambio de categoría migratoria. Éste se refiere, en lo general, a la migración internacional, pues a partir del inicio de la década de los ochenta se ha observado tanto un incremento en la participación de nativos y/o residentes de Baja California en los movimientos emigratorios internacionales, como el surgimiento de nuevas modalidades de migración laboral hacia Estados Unidos que resultan particularmente atractivas para los residentes de la entidad, especialmente a raíz de las modificaciones en la legislación migratoria de Estados Unidos de América en 1986 (i.e. Ley Simpson-Rodino). Es por ello que el crecimiento de la población de Baja California no puede explicarse exclusivamente por el comportamiento de la natalidad, la mortalidad, y de la migración interna, sino que es menester tomar en consideración los movimientos migratorios internacionales para tal caso. La naturaleza de una parte del fenómeno de la emigración hacia Estados Unidos de América dificulta enormemente su caracterización y su cuantificación precisa. Sin embargo, lo que aquí interesa destacar es que en Baja California se dan los dos tipos de migración: interna e internacional, y que estos procesos se encuentran estrechamente relacionados.

\section{DINÁMICA DEMOGRÁFICA DE BAJA CALIFORNIA DURANTE 1985-1990}

En el caso de Baja California, los niveles y las tendencias de los componentes demográficos pueden ser analizados para períodos más recientes que los arriba mencionados, dada la disponibilidad tanto de los resultados de las Encuestas Demográficas de Baja California de 1986 y 1990, como de los resultados definitivos del XI Censo de Población.

El objetivo central de la Encuesta Demográfica de Baja California de 1990 (EDBC90), consistió en actualizar los datos obtenidos en la correspondiente encuesta de 1986 (ver: CONEPOBC-UNAM-UABC:1987), por medio de la réplica de la multicitada encuesta en la que se obtuvieron cuantificaciones sobre migración, mortalidad, fecundidad, vivienda, educación y participación en actividades económicas. En términos de migración, la principal limitante que comparten ambas encuestas consiste en que éstas se elaboraron a partir de un marco muestral de viviendas particulares, lo que implica posibles subestimaciones de los volúmenes de emigración (e.g. cuando todos los residentes de una vivienda emigran), así como de los volúmenes de inmigrantes, puesto que todos aquéllos de estos últimos que residen en viviendas colectivas o institucionales no pueden ser captados a través del marco muestral mencionado. 
A partir de los resultados obtenidos en la encuesta de 1990 y de las estadísticas vitales de Baja California se presenta el cuadro 1, en el que se estiman tanto las existencias como las corrientes de personas con que contaba, y que se presentaron en Baja California y sus municipios durante 1989 (la versión de este cuadro con datos correspondientes a 1986 se puede ver en; Corona, 1991:29).

Las cifras absolutas presentadas en el cuadro 1 permiten advertir, al menos de manera inicial, la magnitud del impacto demográfico que los diferentes flujos de población implican. Al respecto, un primer elemento a notar es el relativo a la magnitud de la emigración desde Baja California, tanto hacia el resto de México como hacia Estados Unidos de América, pues estos movimientos (i.e. 17,107) virtualmente duplicaron el volumen de población que la entidad perdió durante 1989 debido a las defunciones (i.e. 8,791).

Esta relación entre volúmenes de emigración y mortalidad presenta variaciones sustantivas entre los municipios de la entidad. Por ejemplo, mientras que en el caso de Ensenada las tres formas de emigración consideradas (i.e. intraestatal, interestatal e internacional) no logran igualar la reducción de población municipal por defunciones durante 1989, en el caso del municipio de Tijuana la sola emigración hacia Estados Unidos de América es superior a las defunciones registradas durante el año de 1989 , y aquélla tan sólo representa aproximadamente el $60.0 \%$ de la emigración desde Tijuana hacia otras entidades de México.

A la vez, en la contrapartida de esos flujos, también se puede observar que tan sólo la inmigración hacia Baja California desde cl resto de México, superó en los cuatro municipios de la entidad a sus correspondientes cifras de nacimientos registrados durante 1989. Las estimaciones de inmigrantes hacia Baja California durante 1989, al igual que las de emigrantes, permiten advertir variaciones significativas entre los municipios del estado; pues mientras que en Ensenada la inmigración interna y la internacional apenas superan a los nacimientos en ese municipio, en Mexicali los nacimientos representan sólo el $70.0 \%$ de la inmigración interna, en Tecate el $60.0 \%$ y en el municipio de Tijuana sólo el $38.0 \%$ del volumen total de inmigración interna.

Para la entidad en su conjunto, los nacimientos registrados durante 1989 representaron apenas el $53.0 \%$ del flujo total de inmigrantes internos, por lo que una vez considerada la inmigración internacional se puede observar que la inmigración total hacia Baja California durante 1989 logró más que duplicar el número total de nacimientos. Es decir, que las estimaciones de inmigrantes para 1989 permiten observar que la población de la entidad se incrementó por ese concepto en una magnitud que equivaldría al que generaría un nivel de fecundidad dos veces más alto que el prevaleciente en el estado. 
CUADRO 1. Baja California: cuenta demográfica de corrientes y existencias humanas durante 1989, por municipio.

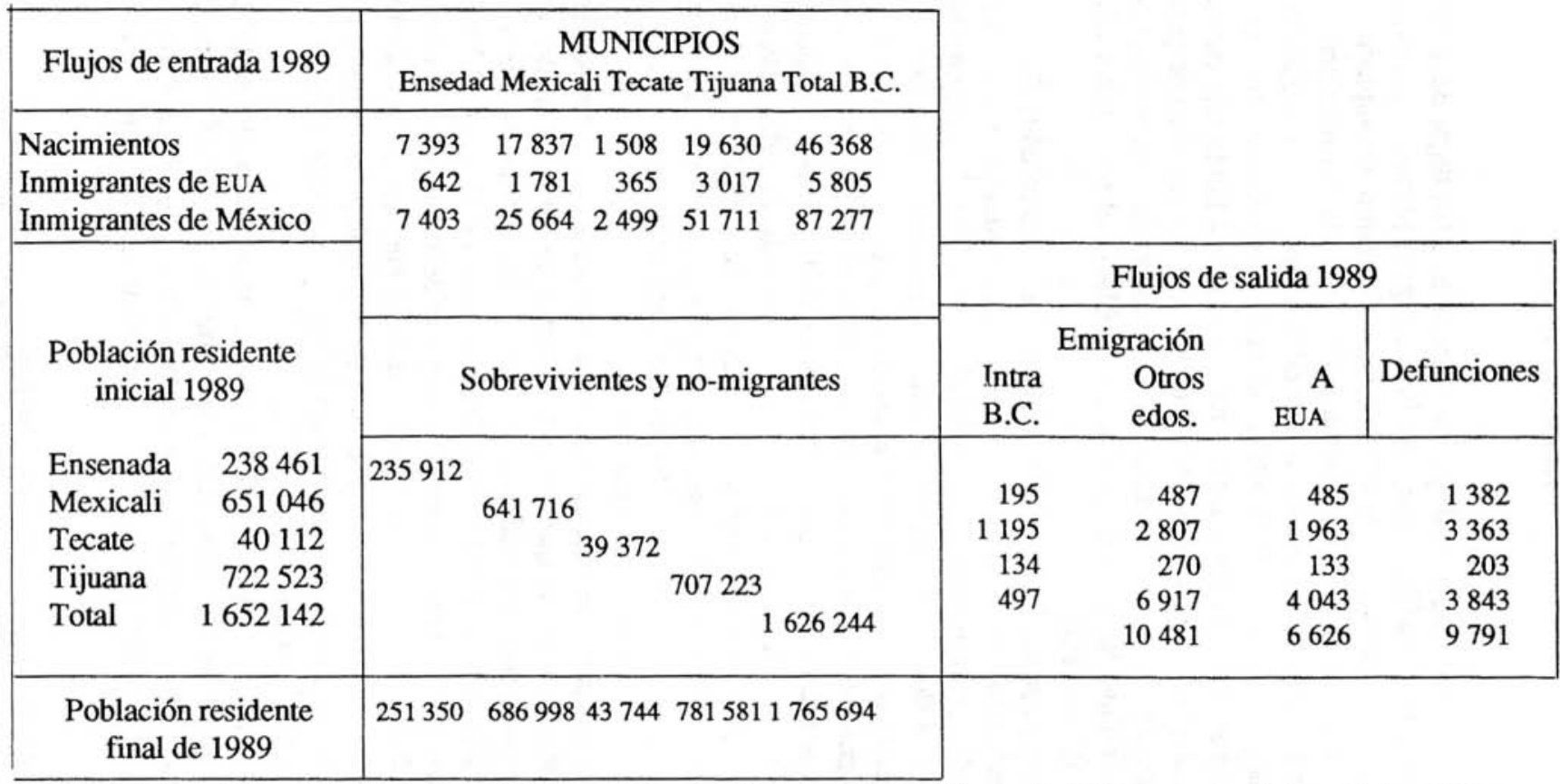

FUENTE: Elaboración propia con base en la Encuesta Demográfica de Baja California de 1990, y en estadísticas vitales del Anuario Estadístico del Estado de Baja California, edición 1991. INEGI. pp. 49-50. 
La elevada magnitud de las estimaciones de los flujos de migración, hacia y desde Baja California, para 1989 obligan a plantearse la posibilidad de que por problemas de diseño, de marco muestral, de implementación de la encuesta o por la combinación de dos o de todas las anteriores causas, nos estuviéramos refiriendo a sobrestimaciones de los volúmenes de población para 1989. Si ése fuera el caso, la sobrestimación resultaría patente al computar las tasas de crecimiento de la población, puesto que en ellas se combinan datos de fuentes independientes. Para despejar esa incógnita y con fines comparativos, a continuación se presenta el cuadro 2 que incluye las tasas de crecimiento poblacional para Baja California entre 1985 y 1990.

Para empezar, los datos presentados en la cuarta columna del cuadro 2 , representados en la figura 1 y que corresponden a la tasa bruta de natalidad (TBN) para los años de 1986, 1989 y 1990, ${ }^{1}$ permiten observar al menos dos elementos importantes. El primero se relaciona con los niveles de la TBN, los que para el estado en su conjunto oscilan alrededor de 27 por millar entre 1986 y 1989 , y se ubica en $25.3 \%$ o durante 1990 . El rango de estas estimaciones, aunado al hecho de que las correspondientes a 1989 se ubican en un punto intermedio entre las de 1986 y las de 1990 - tanto para el estado como para cada uno de los cuatro municipiospermiten señalar que, de estar ante sobrestimaciones de los volúmenes de población para el año de 1989, la TBN correspondiente debería mostrar niveles muy inferiores a los estimados.

Por otra parte, un segundo elemento a destacar es el correspondiente a la tendencia de la TBN que permiten observar los datos del cuadro 2. Esos datos, como pone en evidencia la figura 1, indican un claro proceso de reducción de la natalidad en Baja California durante el período 1986-1990. Esta tendencia de reducción de la TBN que es del orden de 7.7\% entre 1986 y 1990 (i.e. de $27.3 \%$ a $25.2 \%$ o) para la entidad en su conjunto, se reproduce en los municipios de Ensenada, Mexicali y Tecate, mientras que en el caso del municipio de Tijuana la TBN se incrementa de $25.2 \%$ en 1986 a $26.7 \%$ on 1990 (i.e. $6.0 \%$ ).

En la contraparte de los niveles y tendencias observadas en la TBN, la información correspondiente a la tasa bruta de mortalidad (TBM), que se presenta en la quinta columna del cuadro 2 y en la figura 2 , también permite corroborar lo poco probable de estar frente a sobrestimaciones de la

1 La TBN derivada del $x l$ Censo General de Población y Vivienda, corresponde en sentido estricto al período comprendido de marzo de 1989 a marzo de 1990 , y en su cálculo no se realizaron ajustes por mortalidad de menores de un año por no contarse con la información requerida. 
CUADRO 2. Baja California y municipios (1985-1990). Tasas anuales de crecimiento poblacional, por año, entidad y municipios, según composición del crecimiento.

\begin{tabular}{|c|c|c|c|c|c|c|c|}
\hline \multirow{3}{*}{$\begin{array}{c}\text { Año } \\
\text { Entidad } \\
y \\
\text { Municipio }\end{array}$} & \multicolumn{3}{|c|}{$\%$ Anual } & \multicolumn{4}{|c|}{ Composición de las tasas $(\mathrm{o} / \mathrm{o})$} \\
\hline & \multirow{2}{*}{ TCT } & \multirow{2}{*}{$\mathrm{TCN}$} & \multirow{2}{*}{ TCS } & \multicolumn{2}{|c|}{$\mathrm{TCN}$} & \multicolumn{2}{|c|}{ TCS } \\
\hline & & & & TBN & TBM & Inmign. & Emigran. \\
\hline 1986 B.C. & 4.0 & 2.2 & 1.8 & 27.3 & 5.0 & 26.2 & 8.8 \\
\hline Ensenada & 4.3 & 2.7 & 1.6 & 33.0 & 6.0 & 26.4 & 10.5 \\
\hline Mexicali & 2.9 & 2.2 & 0.7 & 27.0 & 4.8 & 16.5 & 10.0 \\
\hline Tecate & 5.0 & 3.3 & 1.7 & 38.2 & 4.9 & 35.8 & 18.7 \\
\hline Tijuana & 4.8 & 2.0 & 2.8 & 25.2 & 4.9 & 39.6 & 11.7 \\
\hline 1989 B.C. & 6.6 & 2.2 & 4.4 & 27.1 & 5.1 & 54.5 & 10.0 \\
\hline Ensenada & 5.3 & 2.5 & 2.8 & 30.2 & 5.6 & 32.8 & 4.8 \\
\hline Mexicali & 5.4 & 2.2 & 3.2 & 26.7 & 5.0 & 41.0 & 8.9 \\
\hline Tecate & 8.7 & 3.1 & 5.5 & 36.0 & 4.8 & 68.3 & 12.8 \\
\hline Tijuana & 7.9 & 2.1 & 5.8 & 26.1 & 5.1 & 72.8 & 15.2 \\
\hline XI CAPyv* B.C. & 3.6 & & & 25.2 & & 37.1 & \\
\hline Ensenada & 4.1 & & & 25.3 & & 33.1 & \\
\hline Mexicali & 1.7 & & & 23.2 & & 20.1 & \\
\hline Tecate & 5.5 & & & 26.5 & & 47.9 & \\
\hline Tijuana & 5.1 & & & 26.7 & & 53.1 & \\
\hline
\end{tabular}

FUENIE: Para 1986: Corona, V. Rodolfo (1991:30); para 1989: Cuadro 1; para 1990: INEGI (1991a:7), INEGI (1991b:2-5, 9-10).

* La TCT, la TBN y la tasa de inmigración calculadas con datos del XI CGPyV, corresponden a los períodos 1980-1990 y 1985-1990, respectivamente.

NOTA: Las tasas de 1986 y 1989 están referidas, para cada municipio y el total de la entidad, al promedio aritmético de las poblaciones residenciales inicial y final del año respectivo. 


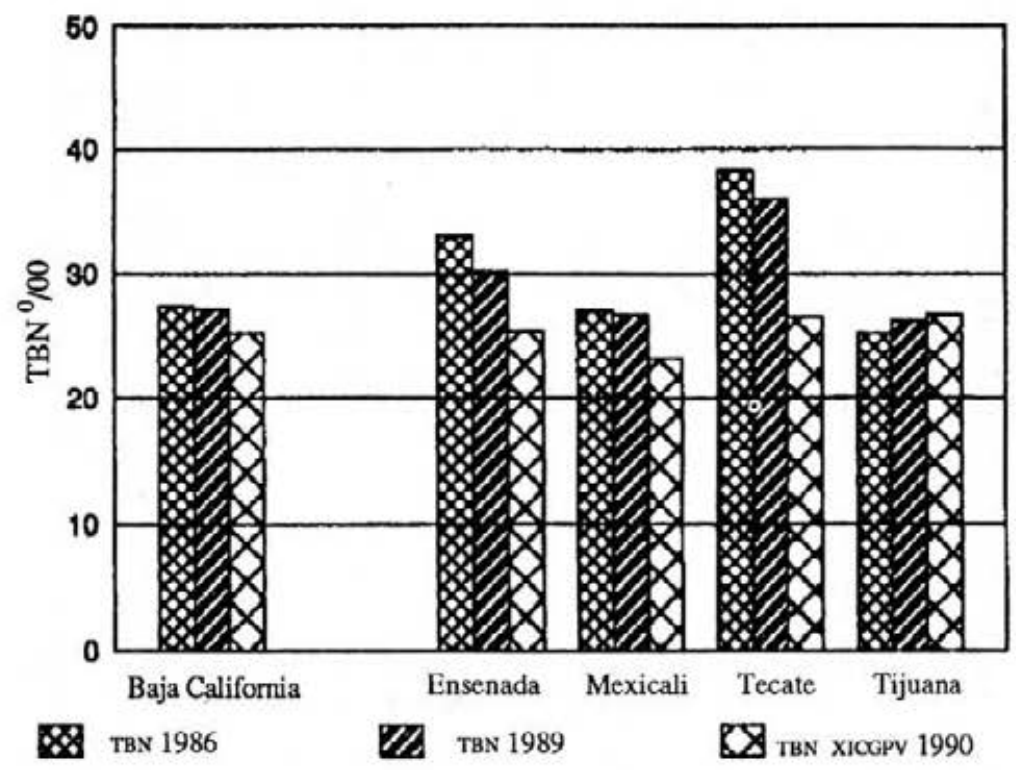

Figura 1. Baja California y municipios 1986-1990, tasas brutas de natalidad $(\%)$. FUENTE: Cuadro 2.

población de 1989. Para los dos años en los que se cuenta con información (i.e. 1986 y 1989), se puede observar que la TBM de Baja California osciló entre 5.0 y 5.1 defunciones por cada mil habitantes.

Las estimaciones de la TBM para 1986 y 1989 , a la vez permiten advertir dos patrones de tendencias en la mortalidad de los municipios del estado. Por una parte, Ensenada y Tecate presentan reducciones en sus niveles de mortalidad del orden de $6.7 \%$ para el primero y $2.0 \%$ para el segundo entre 1986 y 1989. En el caso de Mexicali y Tijuana, por otra parte, se puede observar que las tasas indican incrementos en la mortalidad en estos municipios, pues aquéllas aumentaron aproximadamente $4.0 \%$ en ambos casos, durante el período de 1986 a 1989.

En conjunto, la combinación de las tendencias en las TBN y de las TBM permiten explicar la reducida variación que se observa en la tasa de crecimiento natural (TCN) de la población de Baja California entre 1986 y 1989. En efecto, las TCN, que se presentan en la segunda columna del cuadro 2, indican que a nivel de la entidad en su conjunto no ha habido cambios en el componente natural de su ritmo de crecimiento durante este período. 


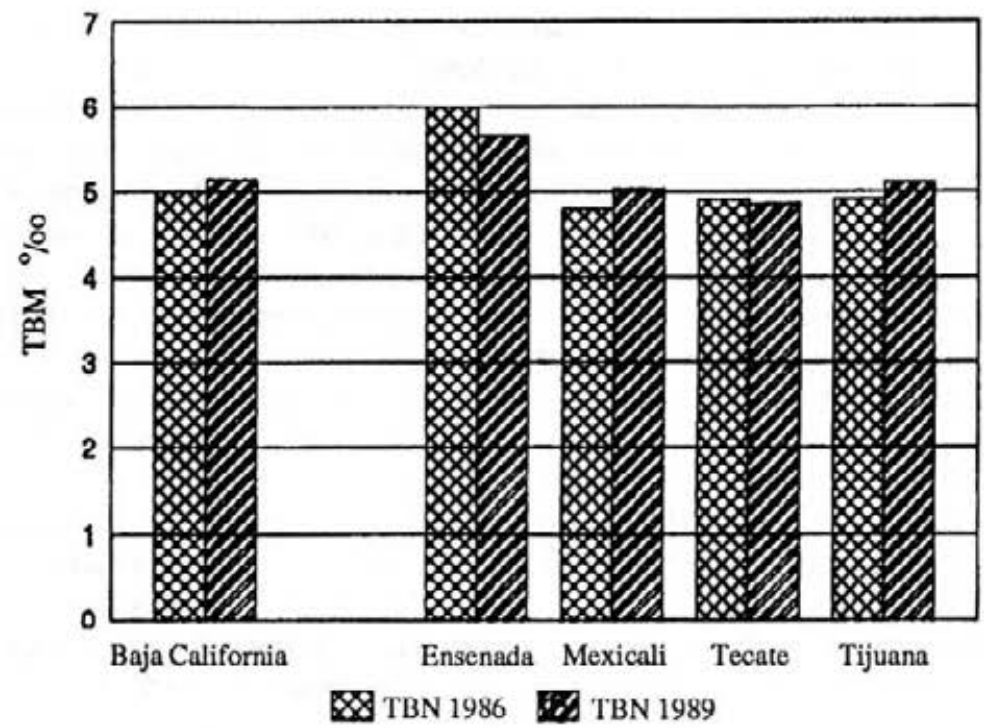

FIGURA 2. Baja California y municipios, tasas brutas de mortalidad 1986 y 1989 ( $\%$ oo). FUENTE: Cuadro 2.

A nivel municipal, sin embargo, se puede observar que, con la excepción de Mexicali donde no hay cambio en la TCN, los demás municipios presentan modificaciones marginales en el nivel de sus TCN. En el caso de Ensenada, la TCN se reduce en $8.0 \%$ de 1986 a 1989; en Tecate el decremento en el crecimiento natural es del orden de $6.1 \%$, y en Tijuana la tendencia es la contraria, pues se observa un incremento en la TCN del orden de $5.0 \%$ entre 1986 y 1989.

Una vez analizados los elementos integrantes del componente natural de la dinámica demográfica de Baja California y habiendo establecido lo poco probable de una sobrestimación de los volúmenes de población para 1989, se abordará la descripción del componente social del crecimiento de la población en Baja california.

Al respecto, el primer punto a señalar es el relativo al notorio incremento en las tasas de crecimiento social que se observa en los datos de la tercera columna del cuadro 2 , tanto para la entidad en su conjunto como para cada uno de los cuatro municipios que la integran. Estos datos, que corresponden al período 1986-1989, indican un revertimiento en la tendencia a la reducción del crecimiento social en Baja California, tendencia en la que venía jugando un 
importante papel la creciente emigración interna e internacional desde cl estado (ver sección I de este documento).

$\mathrm{La}$ información correspondiente a este tipo de movilizaciones de población desde Baja California para los años 1986 y 1989 se presenta en la última columna del cuadro 2, y a partir de ella se pueden hacer al menos dos observaciones. La primera consiste en el hecho de que la tasa de emigración para el total del estado se incrementó de 8.8 por millar a 10.0 por millar durante el período considerado. Sin embargo, la segunda observación necesaria es la que permite advertir que, salvo en el caso del municipio de Tijuana, en los tres municipios restantes la emigración se redujo sustancialmente entre 1986 y 1989 (i.e. $54.3 \%$ en Ensenada; $11.0 \%$ en Mexicali; y, $31.6 \%$ en Tecate).

En la contraparte de las tasas de emigración, los datos presentados en la sexta columna del cuadro 2 y en la figura 3 , permiten establecer el segundo elemento que se encuentra detrás de la reversión de la tendencia de la TCS en Baja California. En este caso nos referimos a las tasas de inmigración que permiten estimar los datos de las tres fuentes que aquí hemos incluido.

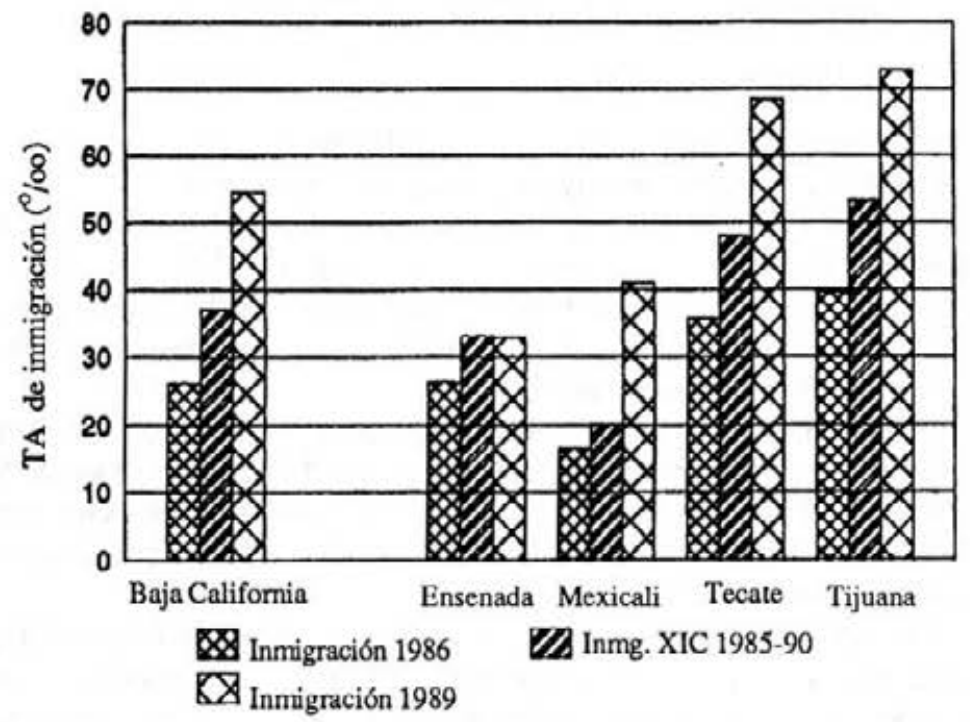

FIGURA 3. Baja California y municipios, tasas anuales de inmigración 1985-1990 (\%o).

FUENTE: Cuadro 2. 
Las tres estimaciones disponibles corresponden a 1986, al período 1985-1990, y al año 1989. De dichas estimaciones y de su representación gráfica se pueden desprender por lo menos dos observaciones de importancia. La primera consiste en que las tres estimaciones identifican volúmenes crecientes de inmigración hacia Baja California para el período de 1985 a 1990, aún consideradas de manera independiente (i.e. 39 mil anuales para 1986; 44 mil por año durante 19851990; y, 87 mil durante 1989).

A la vez, la segunda observación se refiere a la clara tendencia al incremento que describen las estimaciones de inmigración para los tres períodos considerados. Nótese que en este caso, con la excepción marginal para 1989 del municipio de Ensenada, tanto la entidad como todos sus municipios presentan tasas de inmigración que ascienden consistentemente durante el período comprendido de 1985 a 1990.

\section{CONCLUSIONES}

La información hasta aquí presentada, permite derivar las siguientes conclusiones en materia de las tendencias de los componentes de la dinámica demográfica de Baja California para el período 1985-1990:

a) En el rubro de mortalidad del componente natural de la dinámica demográfica de la entidad se ha podido establecer que, a nivel del estado en su conjunto, ésta no ha registrado variaciones significativas en el lapso de 1986 a 1989 (i.e. TBM de 5.0 y $5.1 \%$ o, respectivamente). Sin embargo, mientras los municipios de Ensenada y Tecate han mostrado una continuidad en la tendencia a la reducción de los niveles de la mortalidad durante el período considerado (i.e. reducciones de la TBM de 6.7 y $2.0 \%$, respectivamente), los municipios de Mexicali y Tijuana dan indicios de una reversión en dicha tendencia, al mostrar niveles de mortalidad más elevados en 1989 que en 1986 (i.e. incrementos de la TBM de 4.2 y $4.1 \%$, respectivamente).

b) Para el caso de la natalidad, segundo elemento del componente natural del crecimiento poblacional de Baja California, la información disponible permite advertir claros signos de que, a nivel estatal, la TBN descendió alrededor de $7.7 \%$ en el lapso de 1986 a 1990, pues para ese último año la TBN se ubicó en 25.2 nacimientos por mil habitantes, de acuerdo con la información censal. En este caso se observa que, en tanto que los municipios de Ensenada, Mexicali y Tecate siguen la misma tendencia de reducción de la TBN que la entidad (i.e. con disminuciones de 23.3, 14.1 y $30.6 \%$ en su correspondiente TBN entre 1986 y 1990), en el municipio de Tijuana aparentemente se ha invertido ese patrón, pues la TBN en ese municipio se incrementó de $25.2 \%$ en 1986 a $26.7 \%$ oo en 1990. 
c) En conjunto, los dos elementos anteriores han definido una tendencia de estabilidad en el componente natural de la dinámica demográfica del estado para el período 1986-1989, durante el cual su TCN se ubicó en el orden de $2.2 \%$ anual. A nivel municipal, sin embargo, el patrón de las tendencias del componente natural ha sido heterogéneo, pues mientras que Ensenada y Tecate han reducido sus TCN en 7.4 y $6.1 \%$ en ese período, Mexicali ha permanecido estable en un nivel similar al de la entidad en su conjunto, y Tijuana ha dado indicios de un incremento de aproximadamente $5.0 \%$ en su TCN, la cual de todas formas sigue siendo la más baja de todo el estado, al ubicarse durante 1989 en el nivel de $2.1 \%$ anual.

d) En contrapartida a las tendencias del componente natural de la dinámica poblacional de Baja California, durante el período comprendido de 1986 a 1990 se observa un notable y claro incremento en la movilidad de la población en la entidad. Este proceso de aumento en los flujos de población se ha sustentado en los dos siguientes componentes:

- un incremento en la tasa de emigración del estado en su conjunto (i.e. de 8.8 a $10.0 \%$ entre 1986 y 1989), la cual ha seguido el mismo patrón en el municipio de Tijuana al aumentar de 11.7 a 15.2 $\%$ oo de 1986 a 1989 , pero que ha presentado una tendencia contraria en los tres municipios restantes de la entidad, en donde se observan reducciones sustantivas en las tasas de emigración (i.e. de $54.3 \%$ en Ensenada, $11.0 \%$ en Mexicali, y $31.6 \%$ en Tecate); y,

- una patente y consistente tendencia al incremento en las tasas de inmigración entre 1986 y 1989 , tanto a nivel del estado en su conjunto como en cada uno de los cuatro municipios de Baja California, sobresaliendo en este caso los aumentos entre 1986 y 1989 en los niveles de la tasas de inmigración hacia Mexicali y Tijuana.

e) Se observa pues, en resumen, un renovado peso de componente social en el crecimiento de la población de Baja California durante el período de 1986 a 1989 , en el cual la tasa de crecimiento social —en términos absolutos - involucra el equivalente a dos tantos el crecimiento natural observado en la entidad durante el último de esos dos años. 


\section{BIBLIOGRAFÍA}

CERVERA, F. Miguel. 1991. "La expectativa de población en 1990". En: DEMos. Carta Demografica sobre México. No. 4. UNAM-UNFPAINEGI-COLMEX. México, D.F.

CORONA, V. Rodolfo. 1991. "Algunas características de la dinámica demográfica en la frontera norte de México". Ponencia presentada en: Coloquio sobre Políticas de Población en la Región Frontera Norte de México. SOMEDE-COLEF. Tijuana, B.C. Octubre, 1991. Mimeo.

INEGI. 1991a. XI Censo General de Población y Vivienda, 1990. Baja California, Perfil sociodemográfico. Aguascalientes.

- 1991b. XI Censo General de Población y Vivienda, 1990. Baja California, Resultados Definitivos y Tabulados Básicos. Aguascalientes. 